\title{
Fatores de Vulnerabilidade para o Sofrimento Psíquico Relacionado ao Trabalho de Agentes Administrativos
}

\section{Vulnerability Factors for Psychic Suffering Related to the Work of Administrative Agents}

\author{
Rafael Mondego Fontenele ${ }^{1}$ \\ Hannah Larissa Sousa Leitão ${ }^{2}$ \\ Aline Sharlon Maciel Batista Ramos ${ }^{3}$ \\ Ana Patrícia Fonseca Coelho Galvão ${ }^{4}$ \\ Hariane Freitas Rocha Almeida ${ }^{5}$
}

\section{RESUMO}

Objetivo: identificar fatores de vulnerabilidade para o sofrimento psíquico em agentes administrativos. Metodologia: trata-se de pesquisa descritiva, prospectiva, de caráter quantitativo. Para a coleta dos dados utilizou-se questionário contendo perguntas fechadas elaboradas pelos pesquisadores. A amostra considerou o nível de confiança de $95 \%$ e margem de erro de $5 \%$ e contemplou 249 trabalhadores. A coleta de dados foi realizada no mês de agosto de 2017. Resultados: os fatores de vulnerabilidade para o sofrimento psíquico encontrados no presente estudo foram: o relacionamento com a gestão, classificado como ruim para $53 \%$ da amostra; falta de possibilidade de crescimento dentro da empresa apontada por $69 \%$ dos trabalhadores e a falta de incentivo em busca de qualificação, referida por $73 \%$ dos trabalhadores entrevistados. Conclusão: os dados obtidos ajudam a refletir sobre a importância da implementação de medidas que visam à correção de falhas para reduzir os impactos da jornada de trabalho na saúde física e mental dos trabalhadores, bem como melhorar a qualidade de vida dos mesmos.

\section{DESCRITORES}

Estresse Ocupacional, Estresse Profissional, Estudo Sobre Vulnerabilidade.

\begin{abstract}
Objective: To identify vulnerability factors for psychic suffering in administrative agents. Methodology: This is a descriptive, prospective, quantitative research. For the data collection, a questionnaire containing objective questions elaborated by the researchers, was used. The sample considered the confidence level of $95 \%$ and margin of error of $5 \%$ and included 249 workers. Data collection was performed in august, 2017. Results: the vulnerability factors for psychic suffering found in the present study were: relationship with management, classified as poor for $53 \%$ of the sample; lack of possibility of growth within the company indicated by $69 \%$ of the workers and the lack of incentive in pursuit of qualification, referred by $73 \%$ of the workers interviewed. Conclusion: the data obtained help to reflect on the importance of implementing measures that aim to correct failures to reduce the impacts of the workday on the physical and mental health of workers, as well as improving their quality of life.
\end{abstract}

\section{DESCRIPTORS}

Occupational Stress, Professional Stress, Vulnerability Study.

1 - Mestre em Gestão de Programas e Serviços de Saúde, Universidade CEUMA

2 - Pós-Graduanda em Estratégia Saúde da Família, Faculdade Gianna Beretta.

3 - Doutoranda em Ciências Médicas (Universidade Estadual do Rio de Janeiro). Docente do curso de Enfermagem da Universidade CEUMA.

4 - Mestra em Saúde e Ambiente (Universidade Federal do Maranhão). Docente do curso de Enfermagem da Faculdade Pitágoras São Luís - MA

5 - Pós-Graduanda em Enfermagem do Trabalho, Universidade Cândido Mendes-RJ. 
$\mathrm{O}$ trabalho pode ser entendido através de um universo de significados (sacrifício, sobrevivência, realização, formador da identidade, status social) cujas transformações ocorridas no tempo e na história trazem implicações na vida e na saúde física e mental dos trabalhadores ${ }^{1}$, interferindo diretamente no bem-estar, no modo de trabalhar e, inclusive, na organização coletiva ${ }^{2}$.

Nesta perspectiva, a saúde mental é comumente identificada na saúde do trabalhador como um dos principais fatores responsáveis pela rotatividade, absenteísmo e demissões, pois o trabalho, dependendo da atividade laboral desenvolvida, pode gerar prazer ou sofrimento mental, influenciando diretamente na relação com as demais pessoas envolvidas ${ }^{3}$.

O Burnout é um dos principais sofrimentos psíquicos do trabalhador e caracteriza-se pela cronificação do estresse ocupacional, trazendo consigo consequências negativas tanto em nível individual, como profissional, familiar e social ${ }^{4}$.

$\mathrm{O}$ estado de estresse agudizado pode interferir diretamente na saúde psíquica dos trabalhadores, sendo este percebido na diminuição da qualidade do sono, como por exemplo, em virtude de sobrecarga de atividades, baixos salários e falta de reconhecimento ${ }^{5}$.

O processo de sofrimento psíquico não é, muitas vezes, imediatamente visível. Seu desenvolvimento acontece de forma "silenciosa" ou "invisível", embora também possa eclodir de forma aguda por desencadeada diretamente por fatores presentes no trabalho e configurandose como problema crescente para a atenção à saúde do trabalhador ${ }^{6}$.

O sofrimento psíquico no trabalho está intimamente relacionado com o tipo de ocupação, o tempo de profissão, o tempo na instituição, tipo de cliente, o relacionamento com os colegas no ambiente de trabalho, a pressão sofrida por parte dos superiores (coordenadores, chefes de setor), o ambiente físico (clima, mobília desconfortável), a burocracia nos trâmites de documentos, 0 excesso de normas e protocolos institucionais, a falta de autonomia dentre outras ${ }^{4}$.

Ao se manifestarem acerca da percepção da gênese do desgaste, os trabalhadores apontam como principais fatores a exaustão emocional, elevado para a despersonalização e baixa para a realização pessoal ${ }^{7}$. Estudo sobre sofrimento relacionado ao trabalho evidenciou funcionários que alegam não haver espaço para a criatividade, caracterizando a perda da autonomia e do direito de participar ativamente dos processos, desta forma, as decisões são tomadas sem qualquer participação dos trabalhadores, são decisões tomadas somente pela hierarquia institucional ${ }^{8}$.

Contudo, o sofrimento psíquico no trabalho se apresenta como forma de mal-estar e pode se manifestar através de sintomas como insatisfação, ansiedade, sentimentos de indignidade, de inutilidade, de desvalorização e desgaste no trabalho, além de ultrapassar a esfera mental e somatizar respostas físicas no corpo do trabalhador que se apresentam como sintomas: cefaleias, náuseas, comprometimento da conciliação de sono e repouso, dores abdominais, dores osteomusculares, entre outras ${ }^{9}$.

O déficit de ações que priorizem a saúde dos trabalhadores favorece a ocorrência de agravos à saúde física e mental relacionado ao trabalho, podendo comprometer a qualidade de vida do trabalhador e os resultados esperados pela instituição. Nesse contexto, o presente estudo justifica-se pelo interesse em aprofundar os conhecimentos sobre o tema e pela necessidade de incentivo ao desenvolvimento de medidas de enfrentamento no âmbito individual e coletivo.

Dessa forma, o objetivo do estudo foi identificar fatores de vulnerabilidade para o sofrimento psíquico relacionado ao trabalho de agentes administrativos no Município de São Luís-MA.

\section{Metodologia}

Estudo descritivo, prospectivo de caráter quantitativo, realizado com agentes administrativos dos setores de atendimento de uma instituição de ensino superior em São Luís-MA. A população inicial foi constituída por 700 trabalhadores e após o cálculo amostral, considerando o nível de confiança de $95 \%$ e margem de erro de $5 \%$ reduziu-se a 249 trabalhadores.

Admitiram-se agentes administrativos de ambos os sexos, maiores de 18 anos e que estavam no exercício de suas atividades e excluíram-se os que se encontravam afastados por motivo de doença, licença maternidade ou gozo de férias.

A coleta de dados foi realizada no mês de agosto de 2017 e desenvolvida por meio de duas etapas: a primeira foi a seleção da amostra e convite para participação da pesquisa; e a segunda contava com explicação dos objetivos e 
considerações éticas, e assinatura do termo de consentimento livre e esclarecido.

Para coleta de dados socioeconômicos e demais aspectos importantes para alcance dos objetivos do estudo, aplicou-se um questionário elaborado pelos pesquisadores com base no Negative Acts Questionnaire (NAQ-R) ${ }^{10}$, contendo 20 perguntas fechadas, onde após os esclarecimentos éticos da pesquisa e assinatura do Termo de Consentimento Livre e Esclarecido (TCLE), cada questão devia ser assinalada em apenas uma das alternativas.

Os dados obtidos foram agrupados de acordo com sua especificidade, em função de sua frequência absoluta e dos índices percentuais analisados. Em seguida, foram compilados e apresentados em forma de tabelas elaboradas no programa Microsoft Excel 2010.

O presente estudo obedeceu aos aspectos éticos e legais de pesquisa envolvendo seres humanos, de acordo com as recomendações do Conselho Nacional de Saúde (CNS) e a Resolução 466/2012 11 , foi então submetido à Plataforma Brasil e aprovado pelo Comitê de Ética em Pesquisa da Universidade CEUMA através da emissão do Parecer Consubstanciado de $\mathrm{N}^{\circ} 2.299 .116$.

\section{Resultados}

Na Tabela 1, observa-se a distribuição absoluta e percentual do perfil socioeconômico dos trabalhadores participantes e suas causas de afastamento do trabalho. Com relação ao sexo, observa-se que o grupo é predominado por mulheres $(70 \%)$ e que a idade média está entre 24 e 29 anos (61\%), dos quais 66\% são solteiros e $65 \%$ não têm filhos. Em relação ao grau de escolaridade $41 \%$ possui apenas o ensino médio

Tabela 1. Distribuição absoluta e percentual do perfil dos trabalhadores participantes e suas causas de afastamento. São Luís - MA, 2017.

\begin{tabular}{l|l|c|c}
\hline \multicolumn{1}{c|}{ Categoria } & \multicolumn{1}{c|}{ Variável } & $\mathbf{n}$ & $\%$ \\
\hline Sexo & Masculino & 74 & 30 \\
& Feminino & 175 & 70 \\
\hline Idade & $18-23$ & 26 & 10 \\
& $24-29$ & 152 & 61 \\
& Igual ou acima de 30 & 71 & 29 \\
\hline Estado Civil & Casado & 55 & 22 \\
& Solteiro & 165 & 66 \\
& Outros & 29 & 12 \\
\hline Filhos & 01 filho & 69 & 27 \\
& O2 filhos & 14 & 06 \\
& O3 ou mais filhos & 04 & 02 \\
& Não tem filhos & 162 & 65 \\
\hline Escolaridade & Superior completo & 41 & 17 \\
& Superior incompleto & 95 & 38 \\
& Médio completo & 103 & 41 \\
& Pós-graduação & 10 & 04 \\
\hline Renda Mensal & Até R\$ 1.200,00 & 187 & 75 \\
& De R\$ 1.201,00 e R $\$ 2.500,00$ & 57 & 23 \\
& Acima de 2.501,00 & 05 & 02 \\
\hline Jornada de Trabalho & 40 horas semanais & 46 & 18 \\
& 44 horas semanais & 196 & 79 \\
& Acima de 45 horas & 07 & 03 \\
\hline Total & & $\mathbf{2 4 9}$ & $\mathbf{1 0 0}$ \\
\hline Fonte: Elaborado pelos auto
\end{tabular}


completo. A renda mensal é de até $\mathrm{R} \$ 1.200,00$ $(75 \%)$ e a jornada de trabalho é de 44 horas semanais $(79 \%)$.

Pode-se observar na Tabela 2 a distribuição relativa e percentual da detecção de fatores de vulnerabilidade para o sofrimento psíquico, em relação ao trabalho e ambiente deste. Constatou-se que o frio $(44 \%)$ e o mobiliário inadequado para a postura (19\%) estão entre características presentes no trabalho que favoreceram a experimentação de sentimentos negativos em relação ao trabalho.

Em relação aos sinais e sintomas físicos após a jornada de trabalho, a dor de cabeça $(13 \%)$ e problemas de visão $(38 \%)$ foram mais prevalentes, em contrapartida, $32 \%$ da amostra não apresentou sintomatologia física. Para os sinais e sintomas psíquicos, 9\% apresenta insônia, 20\% apresenta ansiedade, embora, 44\% sentem-se satisfeitos após a jornada de trabalho. Quanto aos sentimentos experimentados ao ter que retornar ao trabalho no dia seguinte, $36 \%$ tem esperança e $30 \%$ não consegue definir.

$\mathrm{Na}$ Tabela 3, pode-se identificar a distribuição numérica e percentual dos fatores de vulnerabilidade para o sofrimento psíquico, relacionados às relações de trabalho e valores organizacionais. Sendo assim, 64\% da amostra tem bom relacionamento com os colegas e $53 \%$ tem um relacionamento ruim com os gestores.

Ao elencar os pontos negativos percebidos no perfil dos gestores, $22 \%$ identificou como autoritarismo, embora, $74 \%$ da amostra destacou que os gestores têm mais qualidade que pontos

Tabela 2. Distribuição absoluta e percentual das características do ambiente laboral, presença de sinais e sintomas físicos e psíquicos pós-jornada de trabalho e sentimentos vivenciados ao saber do retorno ao trabalho no dia seguinte. São Luís - MA, 2017.

\begin{tabular}{l|l|c|c}
\hline Categoria & Variável & $\mathbf{n}$ & \% \\
\hline Características presentes & Ruído & 27 & 11 \\
ambiente de trabalho & Calor & 27 & 11 \\
& Frio & 111 & 44 \\
& Mobília inadequado para postura & 48 & 19 \\
& Materiais de limpeza com cheiros fortes & 14 & 06 \\
& Poeira e sujidade & 22 & 09 \\
\hline Sinais e sintomas físicos & Dores no corpo & 11 & 04 \\
após a jornada de trabalho & LER/DORT & 18 & 07 \\
& Dor de cabeça & 31 & 13 \\
& Dor na coluna & 15 & 06 \\
& Problemas de visão & 95 & 38 \\
& Nenhum & 79 & 32 \\
\hline Sinais e sintomas psíquicos & Tristeza & 03 & 01 \\
após a jornada de trabalho & Insônia & 22 & 09 \\
& Satisfação & 109 & 44 \\
& Falta de apetite & 08 & 03 \\
& Ansiedade & 49 & 20 \\
\hline Sentimentos vivenciados na & Nenhum & 58 & 23 \\
saída do trabalho ao saber & Insatisfação & 02 & 01 \\
que terá que retornar no dia & Alegria & 63 & 25 \\
Seguinte & Insegurança & 10 & 04 \\
& Esperança & 90 & 36 \\
& Raiva & 09 & 04 \\
\hline Total & Não consegue definir & 75 & 30 \\
\hline
\end{tabular}

Fonte: Elaborado pelos autores, 2019. 
Tabela 3. Distribuição absoluta e percentual dos fatores de vulnerabilidade para o sofrimento psíquico, relacionados às relações de trabalho e valores organizacionais. São Luís - MA, 2017.

\begin{tabular}{l|l|c|c}
\hline \multicolumn{1}{c|}{ Categoria } & \multicolumn{1}{c}{ Variável } & $\mathbf{n}$ & $\%$ \\
\hline Relacionamento com os & Rom & 159 & 64 \\
colegas de trabalho & Ruim & 21 & 08 \\
& Muito bom & 67 & 27 \\
& Péssimo & 02 & 01 \\
\hline Relacionamento com os & Rom & 55 & 22 \\
Gestores & Ruim & 132 & 53 \\
& Muito bom & 61 & 24 \\
& Péssimo & 01 & 01 \\
\hline Pontos negativos percebidos & Autoritário & 56 & 22 \\
no perfil dos gestores & Negligente & 04 & 02 \\
& Assédio moral & 04 & 02 \\
& Tem mais qualidades que pontos & 185 & 74 \\
\hline Pontos positivos percebidos & negativos & & \\
no perfil dos gestores & Flexibilidade & 100 & 40 \\
& Inspira confiança & 36 & 14 \\
& Motivador & 94 & 38 \\
& Tem mais pontos negativos que & 19 & 08 \\
\hline Pressão para trabalhar & positivos & & \\
\hline Motivado para o trabalho & Sim & 45 & 18 \\
& Não & 204 & 82 \\
\hline Crescimento na empresa & Sim & 125 & 50 \\
& Não & 124 & 50 \\
\hline Incentivo para a qualificação & Sim & 77 & 31 \\
& Não & 172 & 69 \\
\hline Total & Sim & 68 & 27 \\
\hline Foñ & Não & 181 & 73 \\
\hline
\end{tabular}

Fonte: Elaborado pelos autores, 2019.

negativos. Sobre os pontos positivos no perfil dos gestores, a flexibilidade foi apontada por $40 \%$ da amostra e $38 \%$ para motivação. Em relação a fatores organizacionais, $82 \%$ relatou não sofrer pressão para o trabalho, a amostra ficou dividida entre são motivados e não são motivados para o trabalho, em contrapartida, $69 \%$ não consegue ver possibilidades de crescimento na empresa e $73 \%$ não recebem incentivo para qualificar-se profissionalmente.

\section{Discussão}

Um estudo realizado em grandes empresas do Brasil evidenciou um aumento do número de mulheres no quadro de funcionários nos últimos dois anos. Este fato pode estar relacionado com as questões de gênero, visto que na última década, houve o desenvolvimento de fortes movimentos sociais para que a mulher esteja sempre bem posicionada no mercado de trabalho ${ }^{12}$

Sobre a inserção dos jovens no mercado de trabalho brasileiro no período de 2004 a 2015, destaca-se que houve um crescente aumento das políticas públicas em relação à inserção de jovens no mercado de trabalho, o que pode justificar a representatividade dos jovens na presente estudo ${ }^{13}$.

Em relação à idade, $71 \%$ dos pesquisados têm entre 18 e 29 anos, no entanto, $41 \%$ tem apenas o ensino médio completo e não estão 
cursando o nível superior. Sendo assim, os achados do presente estudo discordam de outra pesquisa que evidenciou um aumento expressivo de pessoas com acesso ao nível superior, baseando-se no crescimento de políticas públicas com a criação do PROUNI (Programa Universidade para Todos), a ampliação de vagas nas Universidades Federais e o programa de cotas, por exemplo ${ }^{14}$.

Sobre o estado civil e relacionamento amoroso, um estudo realizado com jovens entre 18 e 29 anos no Rio de Janeiro, evidenciou que $80 \%$ dos mesmos também eram solteiros, corroborando com os achados no presente estudo em relação à faixa etária ${ }^{15}$.

Sobre a identificação de estresse e agente estressores em trabalhadores do setor administrativo de uma instituição pública de ensino superior, $54,4 \%$ tinha entre um e dois filhos, discordando dos resultados da presente pesquisa cuja maioria dos entrevistados não possuem filhos. Este mesmo estudo também identificou que as baixas remunerações e as longas jornadas de trabalho são consideradas agentes estressores para os trabalhadores. Cabe destacar que a média salarial dos entrevistados no presente estudo estava acima do salário mínimo preconizado no período da coleta de dados e que a jornada de trabalho de 44 horas, ora destacada entre os participantes, também estava devidamente regulamentada pelas leis trabalhistas $^{16}$.

Em relação ao trabalho como determinante do processo saúde-doença, as causas relacionadas com o ambiente físico de trabalho também foram identificadas como fatores de vulnerabilidade para o sofrimento destacandose a temperatura, ruídos entre outros ${ }^{17}$.

Um estudo realizado com trabalhadores do setor administrativo de um hospital universitário em São Paulo evidenciou fatores relacionados à Síndrome da visão do computador em trabalhadores que passavam pelo menos três horas na frente do computador no ambiente de trabalho, corroborando com os resultados encontrados no presente estudo, onde os entrevistados apontaram problemas de visão após a jornada de trabalho ${ }^{18}$.

Considerando os resultados encontrados sobre os sentimentos após a jornada de trabalho, um estudo sobre a satisfação no trabalho e - clima organizacional também identificou que os trabalhadores se sentiam satisfeitos e relacionaram este sentimento com o aumento da produtividade na empresa. No entanto, $20 \%$ da amostra do presente estudo apresentou ansiedade $^{19}$.

Sendo assim, os resultados corroboram com os achados da pesquisa sobre a influência da ansiedade no comportamento humano no ambiente de trabalho, cujo maior contingente de trabalhadores com altos índices de ansiedade foi em profissionais de setores administrativos. A esperança apareceu como um dos principais sentimentos vivenciados antes de retornar ao trabalho, o que pode ser definido como estratégia de enfrentamento e resiliência ${ }^{20}$.

Em estudo realizado em um hospital público da região metropolitana do Porto evidenciou um grau elevado de resiliência nos trabalhadores que participaram da pesquisa, concordando com as estratégias individuais identificadas neste estudo ${ }^{21}$.

O absenteísmo em trabalhadores de um hospital público do Sul do Brasil também foi frequente e os problemas respiratórios também apareceram como uma das principais causas deste tipo de afastamento, identificando 152 trabalhadores que haviam sido afastados do trabalho devido afecções do sistema respiratório, corroborando com a presente pesquisa ${ }^{22}$.

Em recente estudo sobre estresse ocupacional e resiliência no trabalho foi evidenciado que também havia um bom relacionamento entre os colegas de trabalho e que esta boa relação ajudava a minimizar os impactos do estresse relacionado ao ambiente laboral ${ }^{23}$.

$\mathrm{O}$ relacionamento ruim com os gestores e os coordenadores no ambiente de trabalho também foi identificado em uma pesquisa sobre qualidade de vida em trabalhadores do ramo de atendimento a clientes por telefone, o que aumentava o estresse e podia estar relacionado aos adoecimentos físicos e psicológicos dos trabalhadores daquele local ${ }^{24}$.

Em relação aos pontos negativos da gestão, o presente estudo identificou por meio dos entrevistados que existem mais pontos positivos que pontos negativos e isso podem estar relacionados ao fato de também ter sido evidenciada uma gestão flexível. Esta flexibilidade da gestão foi identificada na pesquisa sobre o trabalho de servidores públicos federais, onde destacaram trabalhadores satisfeitos em relação aos seus gestores ${ }^{25}$.

Um estudo sobre vivências de prazer e sofrimento relacionado ao trabalho de agentes 
administrativos de uma agência bancária destacou-se que a pressão por metas e por resultados é um dos principais fatores que leva o trabalhador ao sofrimento relacionado ao trabalho, contudo, os trabalhadores da presente pesquisa não se sentem pressionados para trabalhar ${ }^{26}$.

Ao pesquisarem o bem-estar no trabalho e a percepção de sucesso na carreira em 500 trabalhadores de empresas públicas e privadas no Estado de São Paulo, identificaram que os trabalhadores estavam satisfeitos com as oportunidades de crescimento naquelas empresas, discordando da presente pesquisa, em que os entrevistados não conseguem perceber oportunidades de crescimento ${ }^{27}$.

Sobre o incentivo para a qualificação profissional, a pesquisa sobre a motivação para o desenvolvimento e qualificação profissional em trabalhadores do setor automobilístico em Imperatriz no Estado do Maranhão contrariou os resultados do presente estudo, pois destacou que os trabalhadores se sentiam motivados a buscar qualificação visando crescimento naquela empresa impactando diretamente no alcance de metas $^{28}$.

Diante dos dados obtidos, percebeuse a necessidade de refletir sobre as políticas públicas de saúde no trabalho, bem como a implementação de medidas que diminuam as consequências de fatores como temperatura, ergonomia e outras medidas que reduzam os impactos da jornada de trabalho na saúde física e mental dos trabalhadores.

Cabe destacar a importância da aproximação entre gestores e a equipe de trabalho, visando o fortalecimento das relações e o aumento do incentivo pela qualificação profissional visando possibilidades de promoção na empresa. Ao implementar a correção destas falhas, os impactos serão positivos na qualidade de vida no trabalho e do trabalhador, bem como melhora dos serviços oferecidos e diminuição dos afastamentos por motivo de doenças.

\section{Conclusão}

De acordo com os resultados, o estudo sugere que a origem do sofrimento psíquico relacionado ao trabalho é influenciada por fatores presentes no ambiente de trabalho e pela relação individual de cada trabalhador com estes fatores que podem potencializar o sofrimento psíquico.

Dentre os fatores de vulnerabilidade para o sofrimento psíquico relacionado ao trabalho dos participantes da pesquisa, destacou-se a baixa temperatura, o relacionamento com gestores, a falta de motivação para o trabalho, a ausência de incentivo à qualificação profissional e as baixas perspectivas de crescimento dentro da empresa.

Sendo assim, é imprescindível que os gestores organizacionais realizem as adequações necessárias no ambiente laboral e desenvolvam estratégias que garantam mais qualidade de vida ao trabalhador e contribuam tanto para a redução do presenteísmo e absenteísmo, quanto para o aumento da produtividade e qualidade dos serviços prestados.

Apesar de não se tratar dos objetivos desta pesquisa, mas considerando a possibilidade de desencadear transtornos de ordem maior se ignorados, concluiu-se também que novos estudos deverão ser realizados de modo a aprofundar a investigação dos fatores associados à ocorrência de problemas respiratórios, de modo a contribuir para a adoção de estratégias que proporcionem bem estar, qualidade de vida e maior produtividade a estes trabalhadores.

\section{Referências}

1. Tchiedel RM, Monteiro JK. Prazer e sofrimento no trabaIho das agentes de segurança penitenciária. Estudos de Psicologia. 2013; 18(3):527-535.

2. Silva MP, Bernardo MH, Souza HA. Relação entre saúde mental e trabalho: a concepção de sindicalistas e possíveis formas de enfrentamento. Rev. Bras. Saúde Ocup. [Internet]. 2016 [citado 2019 Maio 02]; 41:e23.

3. Seligmann SE. Trabalho e desgaste mental: o direito de ser dono de si mesmo. $1^{\text {a }}$ edição. Cortez Editora. São Paulo - SP, 2011, 624p.

4. Benevides PAMT (org.). Burnout: quando o trabalho ameaça o bem-estar do trabalhador. $4^{a}$ edição. Casa do Psicólogo. São Paulo, 2010.

5. Galvão APFC, Fontenele RM, Rodrigues ZMR. Stress: fatores situacionais na central de atendimento ao cliente de uma instituição de ensino superior. J Manag Prim Heal Care. 2017; 8(1):27-38.

6. Costa JGF, Medeiros SM. Sofrimento psíquico e trabalho: uma revisão integrativa da literatura. Rev. Bras. Pesq. Saúde. 2013;15(2):116-121. 
7. Portero CS, Vaquero AM. Desgaste profissional, stress e satisfação no trabalho do pessoal de enfermagem em um hospital universitário. Rev. Latino Am. 2015; Enfermagem. 23(3):543-552.

8. Augusto MM, Freitas LG, Mendes AM. Vivências de prazer e sofrimento no trabalho de profissionais de uma fundação pública de pesquisa. Psicologia Revista. 2014; 20(1):34-55.

9. Paula MJ, Moraes AFG. Prazer e sofrimento no trabalho: estudo com terceirizados em um prédio comercial em belo horizonte (MG). Anais do IV Congresso Brasileiro de Estudos Organizacionais - Porto Alegre, RS, Brasil, 19 a 21 de Outubro de 2016.

10. Silva IV, Aquino EML, Pinto ICM. Características psicométricas do Negative Acts Questionnaire para detecção do assédio moral no trabalho: estudo avaliativo do instrumento com uma amostra de servidores estaduais da saúde. Rev. Bras. Saúde Ocup. 2017; 2(42):2.

11. Brasil. Ministério da Saúde. Conselho Nacional de Saúde. Resolução n. 466, de 12 de dezembro de 2012. Aprova diretrizes e normas regulamentadoras de pesquisas envolvendo seres humanos. Brasília, Diário Oficial da União, 12 dez. 2012.

12. Proni TTRW, Proni MW. Discriminação de gênero em grandes empresas no Brasil. Revista Estudos Feministas, 2017, 26(1):e41780.

13. Santos AL, Gimenez DM. Inserção de jovens no mercado de trabalho. Estudos Avançados, 2015, 29(85):153-168.

14. Barros ASX. Expansão da educação superior no Brasil: limites e possibilidades. Educ. Soc. 2015; 36(131):361390.

15. Nascimento BS, Spindola T, Pimentel MRAR, Ramos RCA, Costa RS, Teixeira RS. Comportamento sexual de jovens universitários e o cuidado com a saúde sexual e reprodutiva. Rev Elet Trim Enfermería Global. 2018, 49:248-258.

16. Goulart Junior E, Cardoso HF, Domingues LC, Green RM, Lima TR. Trabalho e estresse: identificação do estrese e dos estressores ocupacionais em trabalhadores de uma unidade administrativa de uma instituição pública de ensino superior (IES). Revista GUAL, Florianópolis, 2014 jan., 7(1):01-17.

17. Cardoso ACM. O trabalho como determinante do processo saúde-doença. Tempo Social, revista de sociologia da USP, 2015, 27(1):73-94.

18. Sá EC. Síndrome da visão do computador e função visual em trabalhadores usuários de computador de um hospital público universitário de São Paulo: prevalência e fatores associados. Saúde, Ética \& Justiça, 2016, 21(2):72-3.
19. Paula APV, Queiroga F. Satisfação no trabalho e clima organizacional: A relação com autoavaliações de desempenho. Rev. Psicol. Organ. Trab. 2015;15(4):362-73.

20. Moraes MCF, Silva NP. Saúde mental e as relações de trabalho: como a ansiedade influencia o comportamento humano no ambiente de trabalho. Revista Interfaces de Saberes. 2015; 14(1):1-16.

21. Silva SM, Borges E, Abreu M, Queirós C, Babtista P, Fell V. Relação entre resiliência e Burnout: promoção da saúde mental e ocupacional dos enfermeiros. Revista Portuguesa de Enfermagem de Saúde Mental. 2016; 16(43).

22. Brey C, Miranda FMD, Haeffner R, Castro IRS, Sarquis LMMS, Felli VE. O absenteísmo entre os trabalhadores de saúde de um hospital público do Sul do Brasil. Revista de Enfermagem do Centro-Oeste Mineiro. 2017; 1(10):7/ e1135.

23. Tereza VFSS, Araujo CCF. Estresse ocupacional e resiliência entre profissionais de saúde. Psicologia: Ciência e Profissão. 2015; 35(3):900-915.

24. Souza DJ, Soares JC, Santos RA, Araújo TCC, Leite TS, Nagamine VHS. O estresse e a qualidade de vida no ambiente de trabalho em organizações do segmento de call center. Psicologia. pt, 2015.

25. Fogaça N, Junior FAC. A hipótese "trabalhador feliz, produtivo": o que pensam os servidores públicos federais. Cad. EBAPE. 2015;13(4):760-775.

26. Máximo TACO, Araújo AJS, Zambroni-de-Souza PC Vivências de prazer e sofrimento no trabalho de gerentes de banco. Psicologia: ciência e profissão, 2014, 34(1):96111.

27. Agapito PR, Filho AP, Siqueira MMM. Bem-estar no trabalho e percepção de sucesso na carreira como antecedentes de intenção de rotatividade. RAM Rev. Adm. Mackenzie. 2015, 16(6):71-93.

28. Conceição MM, Araújo SN, Borges JPS, Rodrigues MS Silva IR. A motivação como fator de influência para o desempenho e qualificação profissional de consultores de vendas externa de uma empresa do setor automobilístico de Imperatriz-MA. Revista de Empreendedorismo e Gestão de Micro e Pequenas Empresas. 2016; 1(2):41-64.

\section{CORRESPONDÊNCIA}

Rafael Mondego Fontenele

Rua Josué Montello, s/n, Renascença II, São Luís-MA, CEP 65-75-120.

E-mail: fhaelmondego@gmail.com 